\title{
Model-Reference Fuzzy Hype-Plane Variable Motor Vibration Control
}

\author{
Abdol Majid Mirshekaran ${ }^{1}$ and Mohsen Imanieh ${ }^{2}$ \\ Department of Electrical and Electronic Engineering, Fasa Branch, Islamic Azad \\ University, Fasa, Iran \\ am82mirshekaran@yahoo.com,imaniehm1960@yahoo.com
}

\begin{abstract}
According to this research paper, fuzzy hype plane controller is applied to robust nonlinear controller to reduce the vibration of motor. To control of multi degree of freedom joint, nonlinear controllers are the best candidate. Sliding mode controller is one of the best choices to robust control of this nonlinear system. The sliding mode controller is used to speed up the error convergence when the error is greater than one. To reduce the error terminal sliding mode controller is recommended in this research. Fuzzy hype-plane variable sliding mode controller is adopted to guarantee the error convergence to zero in a finite time when the error is near the zero. The chattering in the conventional sliding model control systems is avoided with the employed continuous controller. To increase the system robustness in presence of uncertainty fuzzy logic controller is recommended. This technique is used to adjust the band of terminals. The simulation results show that the proposed scheme has strong robust against the uncertainties and disturbances, as well as leads to the convergence of the output to the desired value quickly and precisely than employing either sliding mode controller or terminal sliding mode controller alone.
\end{abstract}

Keywords: Fuzzy hype-plane variable sliding mode controller, fuzzy logic theory, spherical motor, robustness, stability, tuning the terminal

\section{Introduction and Background}

Multi-degrees-of-freedom (DOF) actuators are wide used in a number of Industries. Currently, a significant number of the existing robotic actuators that can realize multi-DOF motion are constructed using gear and linkages to connect several single-DOF motors in series and/or parallel. Not only do such actuators tend to be large in size and mass, but they also have a decreased positioning accuracy due to mechanical deformation, friction and backlash of the gears and linkages. A number of these systems also exhibit singularities in their workspaces, which makes it virtually impossible to obtain uniform, high-speed, and high-precision motion. For high precession trajectory planning and control, it is necessary to replace the actuator system made up of several single-DOF motors connected in series and/or parallel with a single multi-DOF actuator. The need for such systems has motivated years of research in the development of unusual, yet high performance actuators that have the potential to realize multi-DOF motion in a single joint. One such actuator is the spherical motor. Compared to conventional robotic manipulators that offer the same motion capabilities, the spherical motor possesses several advantages [1-2]. Not only can the motor combine 3-DOF motion in a single joint, it has a large range of motion with no singularities in its workspace. The spherical motor is much simpler and more compact in design than most multiple single-axis robotic manipulators. The motor is also relatively easy to manufacture. The spherical motor have potential contributions to a wide range of applications such as 
coordinate measuring, object tracking, material handling, automated assembling, welding, and laser cutting [3]. All these applications require high precision motion and fast dynamic response, which the spherical motor is capable of delivering. Previous research efforts on the spherical motor have demonstrated most of these features. These, however, come with a number of challenges. The spherical motor exhibits coupled, nonlinear and very complex dynamics [4-5]. The design and implementation of feedback controllers for the motor are complicated by these dynamics [6]. The controller design is further complicated by the orientation-varying torque generated by the spherical motor. Some of these challenges have been the focus of previous and ongoing research [7].

Controller (control system) is a device which can sense information from linear or nonlinear system to improve the systems performance and the immune system behavior [89]. In feedback control system considering that there are many disturbances and also variable dynamic parameters something that is really necessary is keeping plant variables close to the desired value. Feedback control system development is the most important thing in many different fields of safety engineering. The main targets in design control systems are safety stability, good disturbance rejection to reach the best safety, and small tracking error[10]. At present, in some applications spherical motors are used in unknown and unstructured environment, therefore strong mathematical tools used in new control methodologies to design nonlinear robust controller with an acceptable safety performance (e.g., minimum error, good trajectory, disturbance rejection). According to the control theory, systems' controls are divided into two main groups: conventional control theory and soft computing control theory. Conventional control theories are work based on manipulator dynamic model. This technique is highly sensitive to the knowledge of all parameters of nonlinear motor's dynamic equation. Conventional control theory is divided into two main groups: linear control theory and nonlinear control theory. Soft computing (intelligent) control theory is free of some challenges associated to conventional control theory. This technique is worked based on intelligent control theory. This theory is divided into the following groups: fuzzy logic theory, neural network theory, genetic algorithm and neuro-fuzzy theory.

To control of this system sliding mode controller is recommend in this research. Sliding mode controller is an influential nonlinear controller to certain and uncertain systems which it is based on system's dynamic model. Sliding mode controller is a powerful nonlinear robust controller under condition of partly uncertain dynamic parameters of system [11]. This controller is used to control of highly nonlinear systems especially for continuum robot. Chattering phenomenon and nonlinear equivalent dynamic formulation in uncertain dynamic parameter are two main drawbacks in pure sliding mode controller [12]. The chattering phenomenon problem in pure sliding mode controller is reduced by using linear saturation boundary layer function but prove the stability is very difficult. Although the fuzzy-logic control is not a new technique, its application in this current research is considered to be novel since it aimed for an automated dynamic-less response rather than for the traditional objective of uncertainties compensation [12]. The intelligent tracking control using the fuzzylogic technique provides a cost-and-time efficient control implementation due to the automated dynamic-less input. This in turn would further inspire multi-uncertainties testing for spherical motor [13].

Although the fuzzy-logic control is not a new technique, its application in this current research is considered to be novel since it aimed for an automated dynamic-less response rather than for the traditional objective of uncertainties compensation[13]. The intelligent tracking control using the fuzzy-logic technique provides a cost-and-time efficient control implementation due to the automated dynamic-less input. This in turn would further inspire multi-uncertainties testing for 3-D motor [11-13]. 
In this research the new technique of sliding mode controller is recommended, namely, hyper-plane sliding mode controller. To modify the response of this controller, on-line tuning terminal sliding mode controller is recommended in this research [14].

This paper is organized as follows; Section 2, is served as an introduction to the dynamic of spherical motor. Part 3, introduces and describes the sliding mode controller, fuzzy logic controller and methodology algorithm. Section 4 presents the simulation results and discussion of this algorithm applied to a spherical motor and the final section describe the conclusion.

\section{Theory}

\section{Dynamic and Kinematics Formulation of Spherical Motor}

Dynamic modeling of spherical motors is used to describe the behavior of spherical motor such as linear or nonlinear dynamic behavior, design of model based controller such as pure sliding mode controller which design this controller is based on nonlinear dynamic equations, and for simulation. The dynamic modeling describes the relationship between motion, velocity, and accelerations to force/torque or current/voltage and also it can be used to describe the particular dynamic effects (e.g., inertia, coriolios, centrifugal, and the other parameters) to behavior of system. Spherical motor is nonlinear and uncertain dynamic parameters and it is 3 degrees of freedom (DOF) electrical motor.

The equation of a spherical motor governed by the following equation:

$$
H(q)\left[\begin{array}{c}
\ddot{\alpha} \\
\ddot{\beta} \\
\ddot{\gamma}
\end{array}\right]+B(q)\left[\begin{array}{c}
\dot{\alpha} \dot{\beta} \\
\dot{\alpha} \dot{\gamma} \\
\dot{\beta} \dot{\gamma}
\end{array}\right]+C(q)\left[\begin{array}{c}
\dot{\alpha}^{2} \\
\dot{\beta}^{2} \\
\dot{\gamma}^{2}
\end{array}\right]=\left[\begin{array}{c}
\tau_{x} \\
\tau_{y} \\
\tau_{z}
\end{array}\right]
$$

Where $\tau$ is actuation torque, $H(q)$ is a symmetric and positive define inertia matrix, $B(q)$ is the matrix of coriolios torques, $\mathrm{C}(\mathrm{q})$ is the matrix of centrifugal torques.

This is a decoupled system with simple second order linear differential dynamics. In other words, the component $\ddot{q}$ influences, with a double integrator relationship, only the variable $q_{i}$, independently of the motion of the other parts. Therefore, the angular acceleration is found as to be:

$$
\ddot{\boldsymbol{q}}=\boldsymbol{H}^{-1}(\boldsymbol{q}) .\{\boldsymbol{\tau}-\{\boldsymbol{B}+\boldsymbol{C}\}\}
$$

This technique is very attractive from a control point of view.

Study of spherical motor is classified into two main groups: kinematics and dynamics. Calculate the relationship between rigid bodies and final part without any forces is called Kinematics. Study of this part is pivotal to design with an acceptable performance controller, and in real situations and practical applications. As expected the study of kinematics is divided into two main parts: forward and inverse kinematics. Forward kinematics has been used to find the position and orientation of task frame when angles of joints are known. Inverse kinematics has been used to find possible joints variable (angles) when all position and orientation of task frame be active [1].

According to the forward kinematics formulation;

$$
\Psi(X, q)=0
$$


Where $\Psi(.) \in R^{n}$ is a nonlinear vector function, $X=\left[X_{1}, X_{2}, \ldots \ldots, X_{l}\right]^{T}$ is the vector of task space variables which generally task frame has three task space variables, three orientation, $q=\left[q_{1}, q_{2}, \ldots, q_{n}\right]^{T}$ is a vector of angles or displacement, and finally $n$ is the number of actuated joints. The Denavit-Hartenberg (D-H) convention is a method of drawing spherical motor free body diagrams. Denvit-Hartenberg (D-H) convention study is necessary to calculate forward kinematics in this motor.

A systematic Forward Kinematics solution is the main target of this part. The first step to compute Forward Kinematics (F.K) is finding the standard D-H parameters. The following steps show the systematic derivation of the standard D-H parameters.

1. Locate the spherical motor

2. Label joints

3. Determine joint rotation $(\theta)$

4. Setup base coordinate frames.

5. Setup joints coordinate frames.

6. Determine $\alpha_{i}$, that $\alpha_{i}$, link twist, is the angle between $Z_{i}$ and $Z_{i+1}$.

7. Determine $d_{i}$ and $a_{i}$, that $a_{i}$, link length, is the distance between $Z_{i}$ and $Z_{i+1}$ along $X_{i}$. $d_{i}$, offset, is the distance between $X_{i-1}$ and $X_{i}$ along $Z_{i}$ axis.

8. Fill up the D-H parameters table. The second step to compute Forward kinematics is finding the rotation matrix $\left(R_{n}^{0}\right)$. The rotation matrix from $\left\{F_{i}\right\}$ to $\left\{F_{i-1}\right\}$ is given by the following equation;

$$
R_{i}^{i-1}=U_{i\left(\theta_{i}\right)} V_{i\left(\alpha_{i}\right)}
$$

Where $U_{i\left(\theta_{i}\right)}$ is given by the following equation;

$$
U_{i\left(\theta_{i}\right)}=\left[\begin{array}{ccc}
\cos \left(\theta_{i}\right) & -\sin \left(\theta_{i}\right) & 0 \\
\sin \left(\theta_{i}\right) & \cos \left(\theta_{i}\right) & 0 \\
0 & 0 & 1
\end{array}\right]
$$

and $V_{i\left(\alpha_{i}\right)}$ is given by the following equation;

$$
V_{i\left(\theta_{i}\right)}=\left[\begin{array}{ccc}
1 & 0 & 0 \\
0 & \cos \left(\alpha_{i}\right) & -\sin \left(\alpha_{i}\right) \\
0 & \sin \left(\alpha_{i}\right) & \cos \left(\alpha_{i}\right)
\end{array}\right]
$$

So $\left(R_{n}^{0}\right)$ is given by [8]

$$
R_{n}^{0}=\left(U_{1} V_{1}\right)\left(U_{2} V_{2}\right) \ldots \ldots\left(U_{n} V_{n}\right)
$$

The final step to compute the forward kinematics is calculate the transformation ${ }_{n}^{0} T$ by the following formulation [3]

$$
{ }_{n}^{0} T={ }_{1}^{0} T \cdot{ }_{2}^{1} T \cdot{ }_{3}^{2} T \ldots \ldots . .{ }_{n}^{n-1} T=\left[\begin{array}{cc}
R_{n}^{0} & 0 \\
0 & 1
\end{array}\right]
$$




\section{Methodology}

A significant challenge in control algorithms is a linear behavior controller design for nonlinear systems. When system works with various parameters and hard nonlinearities this technique is very useful in order to be implemented easily but it has some limitations such as working near the system operating point. Some of nonlinear systems which work in industrial processes are controlled by linear PID controllers, but the design of linear controller for spherical motors are extremely difficult because they are nonlinear, uncertain and MIMO. To reduce above challenges the nonlinear robust controllers is used to systems control. One of the powerful nonlinear robust controllers is sliding mode controller (SMC), although this controller has been analyzed by many researchers but the first proposed was in the 1950.This controller is used in wide range areas such as in robotics, in control process, in aerospace applications and in power converters because it has an acceptable control performance and solve some main challenging topics in control such as resistivity to the external disturbance. The lyapunov formulation can be written as follows,

$$
V=\frac{1}{2} S^{T} . H . S
$$

The derivation of $V$ can be determined as,

$$
\dot{V}=\frac{1}{2} S^{T} \cdot \dot{H} \cdot S+S^{T} H \dot{S}
$$

The dynamic equation of spherical motor can be written based on the sliding surface as

$$
H \dot{S}=-V S+H \dot{S}+V S-\tau
$$

It is assumed that

$$
S^{T}(\dot{H}-2 V) S=0
$$

by substituting (11) in (10)

$$
\dot{V}=\frac{1}{2} S^{T} \dot{H} S-S^{T} V S+S^{T}(H \dot{S}+V S-\tau)=S^{T}(H \dot{S}+V S-\tau)
$$

Suppose the control input is written as follows

$$
\widehat{\boldsymbol{\tau}}=\widehat{\boldsymbol{\tau}_{\text {eq }}}+\widehat{\boldsymbol{\tau}_{\text {dls }}}=\left[\widehat{\boldsymbol{H}^{-1}}(\widehat{\boldsymbol{V}})+\dot{S}\right] \widehat{H}+K \cdot \operatorname{sgn}(S)+K_{v} S
$$

By replacing the equation (14) in (13)

$$
\begin{aligned}
& \dot{V}=S^{T}\left(H \dot{S}+V S-\widehat{H} \dot{S}-\widehat{V} S-K_{v} S-K \operatorname{sgn}(S)=S^{T}\left(H \dot{S}+\widetilde{V} S-K_{v} S-\right.\right. \\
& \operatorname{Ksgn}(S))
\end{aligned}
$$

It is obvious that 


$$
\left|\widetilde{H} \dot{S}+\widetilde{V} S-K_{v} S\right| \leq|\widetilde{H} \dot{S}|+|\widetilde{V} S|+\left|K_{v} S\right|
$$

The Lemma equation in spherical motor system can be written as follows

$$
K_{u}=\left[|\widetilde{H} \dot{S}|+|V S|+\left|K_{v} S\right|+\eta\right]_{i}, i=1,2,3,4, \ldots
$$

The equation (12) can be written as

$$
K_{u} \geq\left|\left[H \dot{S}+V S-K_{v} S\right]_{i}\right|+\eta_{i}
$$

Therefore, it can be shown that

$$
\dot{V} \leq-\sum_{i=1}^{n} \eta_{i}\left|S_{i}\right|
$$

Based on above discussion, the control law for spherical motor is written as:

$$
\boldsymbol{U}=\boldsymbol{U}_{\text {eq }}+\boldsymbol{U}_{\text {swith }}
$$

Where, the model-based component $\boldsymbol{U}_{\boldsymbol{e q}}$ is the nominal dynamics of systems and $\boldsymbol{U}_{\boldsymbol{e q}}$ can be calculate as follows:

$$
U_{e q}=\left[H^{-1}(B+C)+\dot{S}\right] H
$$

$\boldsymbol{U}_{\text {SWITCH}}$ is computed as;

$$
U_{\text {switch }}=K \cdot \operatorname{SGN}(\lambda e+\dot{e})
$$

by replace the formulation (22) in (20) the control output can be written as;

$$
U=U_{e q}+K . S G N(S)
$$

By (23) and (21) the sliding mode control of spherical motor is calculated as;

$$
U=\left[H^{-1}(B+C)+\dot{S}\right] H+K \cdot \operatorname{SGN}(S)
$$

Figure 1 shows the conventional sliding mode controller for three dimension of spherical motor. 


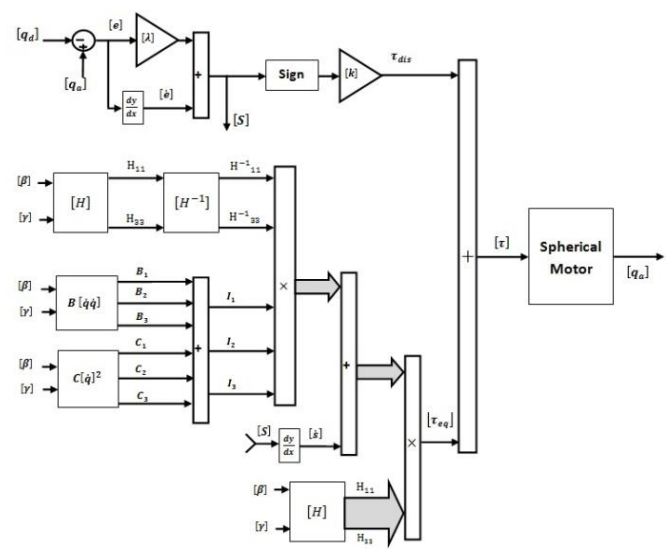

Figure 1. Sliding Mode Control: 3 DOF Spherical Motor

For the terminal sliding mode control part, the hype-plane is defined as

$S=\lambda \cdot e^{P}+\dot{e}$

Where $0<p<1$

Supposed that $U$ is the universe of discourse and $x$ is the element of $U$, therefore, a crisp set can be defined as a set which consists of different elements $(x)$ will all or no membership in a set. A fuzzy set is a set that each element has a membership grade, therefore it can be written by the following definition;

$A=\left\{x, \mu_{A}(x) \mid x \in X\right\} ; A \in U$

Where an element of universe of discourse is $x, \mu_{A}$ is the membership function (MF) of fuzzy set. The membership function $\left(\mu_{A}(x)\right)$ of fuzzy set $A$ must have a value between zero and one. If the membership function $\mu_{A}(x)$ value equal to zero or one, this set change to a crisp set but if it has a value between zero and one, it is a fuzzy set. Defining membership function for fuzzy sets has divided into two main groups; namely; numerical and functional method, which in numerical method each number has different degrees of membership function and functional method used standard functions in fuzzy sets. The membership function which is often used in practical applications includes triangular form, trapezoidal form, bell-shaped form, and Gaussian form.

Linguistic variable can open a wide area to use of fuzzy logic theory in many applications (e.g., control and system identification). In a natural artificial language all numbers replaced by words or sentences.

If - then Rule statements are used to formulate the condition statements in fuzzy logic. A single fuzzy If - then rule can be written by

\section{If $x$ is $A$ Then $y$ is $B$}

where $A$ and $B$ are the Linguistic values that can be defined by fuzzy set, the If - part of the part of " $x$ is $A$ " is called the antecedent part and the then - part of the part of " $y$ is $B$ " is called the Consequent or Conclusion part. The antecedent of a fuzzy if-then rule can have multiple parts, which the following rules shows the multiple antecedent rules: 
where $e$ is error, $\dot{e}$ is change of error, $N B$ is Negative Big, $M L$ is Medium Left, $T$ is torque and $L L$ is Large Left. If - then rules have three parts, namely, fuzzify inputs, apply fuzzy operator and apply implication method which in fuzzify inputs the fuzzy statements in the antecedent replaced by the degree of membership, apply fuzzy operator used when the antecedent has multiple parts and replaced by single number between 0 to 1 , this part is a degree of support for the fuzzy rule, and apply implication method used in consequent of fuzzy rule to replaced by the degree of membership. The fuzzy inference engine offers a mechanism for transferring the rule base in fuzzy set which it is divided into two most important methods, namely, Mamdani method and Sugeno method. Mamdani method is one of the common fuzzy inference systems and he designed one of the first fuzzy controllers to control of system engine. Mamdani's fuzzy inference system is divided into four major steps: fuzzification, rule evaluation, aggregation of the rule outputs and defuzzification. Michio Sugeno use a singleton as a membership function of the rule consequent part. The following definition shows the Mamdani and Sugeno fuzzy rule base

$\begin{array}{lccccc}\text { Mamdani } & F . R^{1}: \text { if } & x \text { is A and } & y \text { is } B & \text { then } & z \text { is } C \\ \text { Sugeno } & F . R^{1}: \text { if } & x \text { is A and } & y \text { is } B & \text { then } & f(x, y) \text { is } C\end{array}$

When $x$ and $y$ have crisp values fuzzification calculates the membership degrees for antecedent part. Rule evaluation focuses on fuzzy operation $(A N D / O R)$ in the antecedent of the fuzzy rules. The aggregation is used to calculate the output fuzzy set and several methodologies can be used in fuzzy logic controller aggregation, namely, Max-Min aggregation, Sum-Min aggregation, Max-bounded product, Max-drastic product, Maxbounded sum, Max-algebraic sum and Min-max. Two most common methods that used in fuzzy logic controllers are Max-min aggregation and Sum-min aggregation. Max-min aggregation defined as below

$$
\mu_{U}\left(x_{k}, y_{k}, U\right)=\mu_{\cup_{i=1}^{r} F R^{i}}\left(x_{k}, y_{k}, U\right)=\max \left\{\min _{i=1}^{r}\left[\mu_{R}{ }_{p q}\left(x_{k}, y_{k}\right), \mu_{p_{m}}(U)\right]\right\}
$$

The Sum-min aggregation defined as below

$$
\mu_{U}\left(x_{k}, y_{k}, U\right)=\mu_{\cup_{i=1}^{r} F R^{i}}\left(x_{k}, y_{k}, U\right)=\sum \min _{i=1}^{r}\left[\mu_{R p q}\left(x_{k}, y_{k}\right), \mu_{p_{m}}(U)\right]
$$

where $r$ is the number of fuzzy rules activated by $x_{k}$ and $y_{k}$ and also $\mu_{\cup_{i=1}^{r} F R^{i}}\left(x_{k}, y_{k}, U\right)$ is a fuzzy interpretation of $i-t h$ rule. Defuzzification is the last step in the fuzzy inference system which it is used to transform fuzzy set to crisp set. Consequently defuzzification's input is the aggregate output and the defuzzification's output is a crisp number. Centre of gravity method (COG) and Centre of area method (COA) are two most common defuzzification methods, which $C O G$ method used the following equation to calculate the defuzzification

$\operatorname{COG}\left(x_{k}, y_{k}\right)=\frac{\sum_{i} U_{i} \sum_{j=1}^{r} \cdot \mu_{u}\left(x_{k}, y_{k}, U_{i}\right)}{\sum_{i} \sum_{j=1}^{r} \cdot \mu_{u}\left(x_{k}, y_{k}, U_{i}\right)}$ 
and $C O A$ method used the following equation to calculate the defuzzification

$\operatorname{COA}\left(x_{k}, y_{k}\right)=\frac{\sum_{i} U_{i} \cdot \mu_{u}\left(x_{k}, y_{k}, U_{i}\right)}{\sum_{i} \mu_{U}\left(x_{k}, y_{k}, U_{i}\right)}$

Where $\operatorname{COG}\left(x_{k}, y_{k}\right)$ and $\operatorname{COA}\left(x_{k}, y_{k}\right)$ illustrates the crisp value of defuzzification output, $U_{i} \in U$ is discrete element of an output of the fuzzy set, $\mu_{U} \cdot\left(x_{k}, y_{k}, U_{i}\right)$ is the fuzzy set membership function, and $r$ is the number of fuzzy rules.

Based on foundation of fuzzy logic methodology; fuzzy logic controller has played important rule to design nonlinear controller for nonlinear and uncertain systems. However the application area for fuzzy control is really wide, the basic form for all command types of controllers consists of;

- Input fuzzification (binary-to-fuzzy[B/F]conversion)

- Fuzzy rule base (knowledge base)

- Inference engine

- Output defuzzification (fuzzy-to-binary[F/B]conversion).

Figure 2 shows fuzzy controller operation.

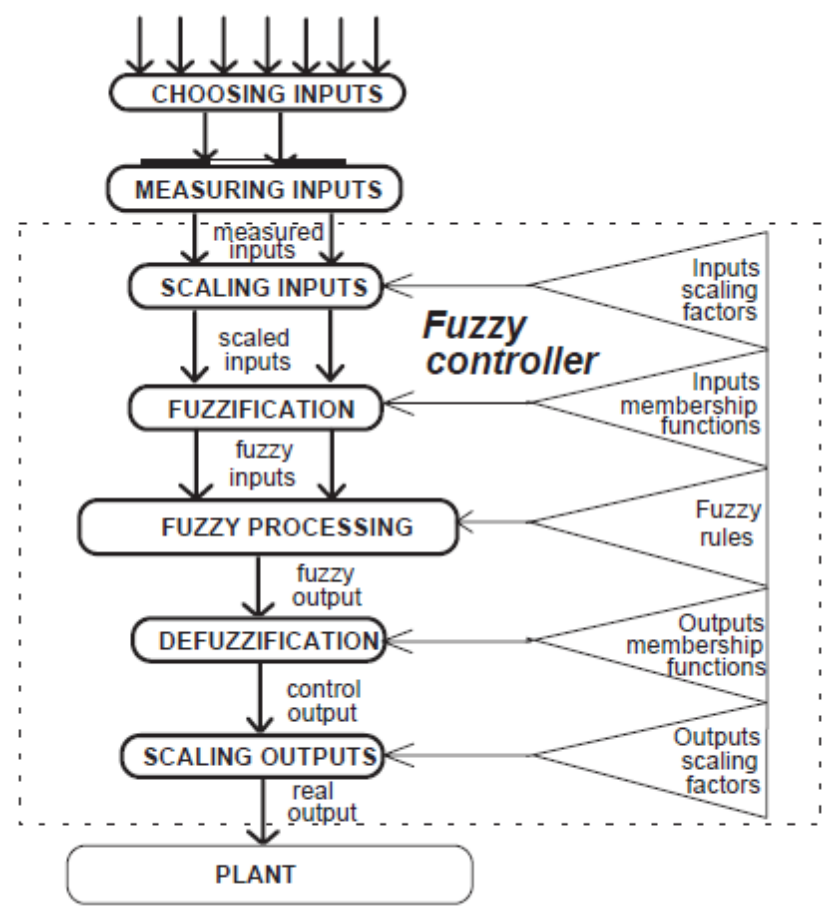

Figure 2. Fuzzy Controller Operation

The new fuzzy hype-plane variable can be written as:

$S=\alpha . S_{T}+(1-\alpha) . S=\alpha\left(\lambda . e^{P}+\dot{e}\right)+(1-\alpha) .(\lambda e+\dot{e}) \dot{e}+\lambda \alpha e^{P}+$

$\lambda(1-\alpha) e$

And $\alpha$ is fuzzy output. 


\section{Result and Discussion}

Fuzzy hype-plane variable sliding mode controller and conventional sliding mode controller are tested to Step response trajectory. The simulation was implemented in MATLAB/SIMULINK environment. These systems are tested by band limited white noise with a predefined $30 \%$ of relative to the input signal amplitude. This type of noise is used to external disturbance in continuous and hybrid systems and applied to nonlinear dynamic of these controllers.

Trajectory follow: Figure 3 shows the trajectory performance in fuzzy hype plane sliding mode controller and conventional sliding mode controller. Due to following graph SMC has moderate chattering but proposed method can eliminate it.

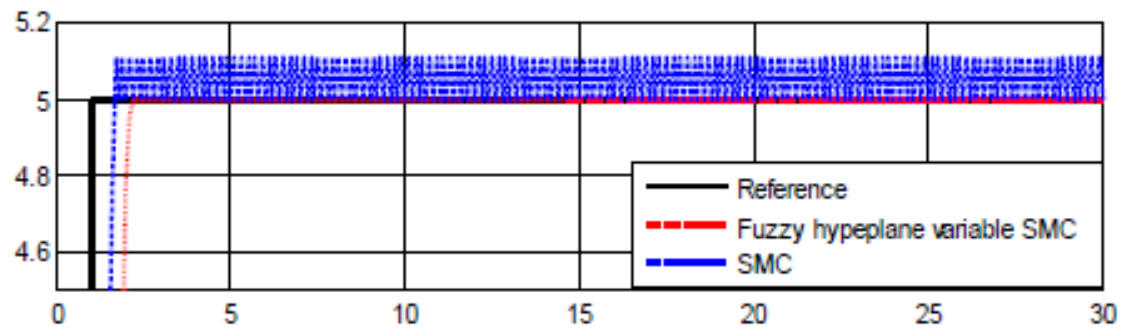

Figure 3. Trajectory Follows: Fuzzy Hype-plane Variable SMC vs. SMC

Disturbance trajectory follows: Figure 4 shows the power disturbance elimination in proposed method and pure sliding mode controller. The disturbance rejection is used to test and analyzed the robustness comparisons of these controllers for step trajectory. A band limited white noise with predefined of $30 \%$ the power of input signal value is applied to the step trajectory. It found fairly fluctuations in SMC trajectory responses. According to the following graph, pure SMC has moderate chattering in presence of external disturbance and uncertainty. However SMC has moderate chattering but this type of controller is robust. Fuzzy hype-plane variable SMC can eliminate the chattering and fluctuation in presence of uncertainty and external disturbance, therefore this type of controller is a robust.

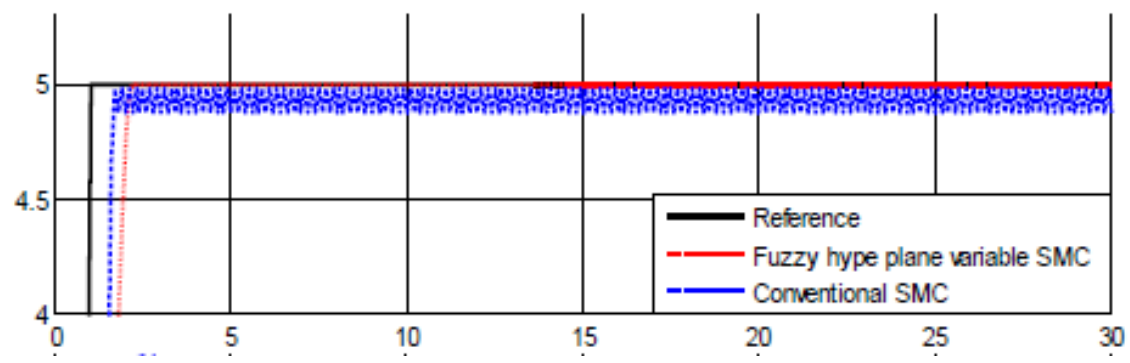

Figure 4. Disturbance Trajectory Follows: Fuzzy Hype-plane Variable SMC vs. SMC

According to above two Figures (Figure 3 and Figure 4) fuzzy hype plane variable SMC and SMC are robust but SMC has chattering and proposed method is used to improve the chattering as well as improve the robustness. 


\section{Conclusion}

In this research two objectives are very important to control of spherical motor: the first one is eliminate the chattering and the second one is improving the robustness. To reach to above two objectives fuzzy hyper-plane variable sliding mode controller is recommend with application to spherical motor.

In this type of method the sliding surface improved by hyper-plane methodology. In this method sliding surface is divided into two main parts: the linear sliding mode part and the terminal sliding mode part. Fuzzy logic controller is used to improve the system quality and estimate the system dynamic in uncertain condition.

According to simulation result, this type of controller is stable and robust against uncertainty and external disturbance and has a better performance than conventional SMC.

\section{References}

[1] G. I. Vachtsevanos, K. Davey and K. M. Lee, "Development of a Novel Intelligent Robotic Manipulator”, IEEE Control System Magazine, (1987), pp. 9-15.

[2] K. Davey, G. I. Vachtsevanos and R. Powers, "An analysis of Fields and Torques in Spherical Induction Motors”, IEEE Transactions on Magnetics, vol. MAG-23, (1987), pp. 273-282.

[3] A. Foggia, E. Oliver and F. Chappuis, "New Three Degrees of Freedom Electromagnetic Actuator", Conference Record -lAS Annual Meeting, vol. 35, (1988), New York.

[4] K. M. Lee, G. Vachtsevanos and C-K. Kwan, "Development of a Spherical Wrist Stepper Motor", Proceedings of the 1988 IEEE International Conference on Robotics and Automation, Philadelphia, PA. April 26-29.

[5] K. M. Lee and I. Pei, "Kinematic Analysis of a Three Degree-of-Freedom Spherical Wrist Actuator", The Fifth International Conference on Advanced Robotics, Italy, (1991).

[6] I. Wang, G. Jewel and D. Howe, "Modeling of a Novel Spherical Pennanent Magnet Actuator", Proceedings of IEEE International Conference on Robotics and Automation, Albuquerque, New Mexico, (1997), pp. 1190-1195.

[7] I. Wang, G. Jewel and D. Howe, "Analysis, Design and Control of a Novel Spherical Pennanent Magnet Actuator", IEE Proceedings on Electrical Power Applications", vol. 154, no. 1, (1998).

[8] G. S. Chirikjian and D. Stein, "Kinematic Design and Commutation of a Spherical Stepper Motor", IEEEIASME Transactions on Mechatronics, vol. 4, no. 4, Piscataway, New Jersey, (1999) December, pp. 342-353.

[9] K. Kahlen and R. W. De Doncker, "CW'l'ent Regulators for Multi-phase Pennanent Magnet Spherical Machines", Industry Applications Conference Record of the 2000 IEEE, vol. 3, (2000), pp. 2011-2016.

[10] K. M. Lee, I. Pei and U. Gilboa, "On the Development of a Spherical Wrist Actuator," Proceedings of the 16th NSF Conference on Manufacturing Systems Research, Tempe AZ, (1990) January 8-12.

[11] C. Yang and Y. S. Back, "Design and Control of the 3-dcgn:es of freedom actuator by Controlling the Electromagnetic Force”, IEEE Transactions on Magnetics, (1999) May, pp. 3607-3609.

[12] M. A. Tayebi, F. Piltan, M. Piltan, M. Yaghoot and M. Esmaeili, "Design New Intelligent-Base Chattering Free Nonlinear Control of Spherical Motor", International Journal of Intelligent Systems and Applications, vol. 6, no. 10, (2014), pp. 55-66. DOI: 10.5815/ijisa.2014.10.08. (DOAJ, DOI: 10.5815)

[13] M. Yaghoot, F. Piltan, M. Esmaeili, M. A. Tayebi and M. Piltan,'Design Intelligent Robust Model-base Sliding Guidance Controller for Spherical Motor", IJMECS, vol. 6, no. 3, pp. 61-72, (2014). DOI:10.5815/ijmecs.2014.03.08

[14] F. Matin, F. Piltan, H. Cheraghi, N. Sobhani and M. Rahmani, "Design Intelligent PID like Fuzzy Sliding Mode Controller for Spherical Motor”, IJIEEB, vol. 6, no. 2, pp. 53-63, (2014). DOI:10.5815/ijieeb.2014.02.07 


\section{Authors}

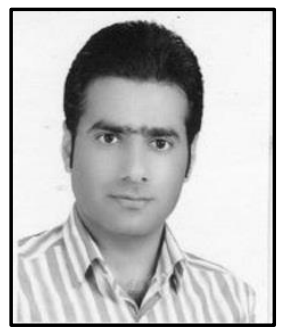

Abdol Majid Mirshekaran, he is currently working as a co researcher in Control and Robotic Lab at the Islamic Azad University. He is a Master in field of Electrical Engineering from Islamic Azad University, IRAN. His current research interests are in the area of nonlinear control, artificial control system and robotics, and spherical motor.

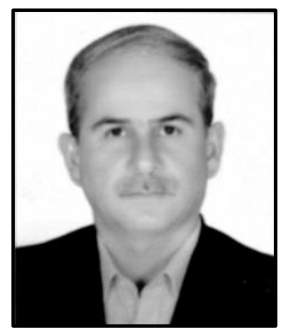

Dr. Mohsen Imanieh, he graduated from the University of Salford, UK, in 1989. He worked as a research fellow from 1985 to 1992 at Salford University. He was awarded a $\mathrm{PhD}$ in the field of crystal growth of $\mathrm{CuInGaSe} 2$ used for the substrates of solar cells. He is currently working as a member of the academic staff at The Azad University of Fasa, Iran, in the Department of Electronics. 